\title{
Resynchronization in Heart Failure: What to choose?
}

\author{
Mohammad El Baba ${ }^{1}$, Moses Wananu ${ }^{1}$, Marwan Refaat ${ }^{2}$, and Jayakumar Sahadevan ${ }^{1}$ \\ ${ }^{1}$ University Hospitals of Cleveland \\ ${ }^{2}$ American University of Beirut Medical Center
}

June 3, 2021

\begin{abstract}
Achieving Cardiac resynchronization therapy (CRT) with Biventricular pacing(BiVP) pacing for patients with moderate-tosevere heart failure (HF), left ventricular (LV) systolic dysfunction and ventricular dyssynchrony is well established and is currently the standard of care. Multiple studies have demonstrated significant improvement in quality of life, functional status, and exercise capacity in patients with New York Heart Association (NYHA) class III and IV heart failure who underwent resynchronization therapy 1,2. In addition, resynchronization therapy is associated with survival benefit3. However, one third of patients do not respond to BIVP. New modalities for resynchronization have emerged namely His bundle pacing (HBP) and left ventricular septal pacing (LVSP). In this paper, we will review the benefits and limitations of BiVP and also the role of new pacing modalities such as HBP and LVSP in patients with HF with reduced left ventricular ejection fraction (LVEF) and electrical dysynchrony.
\end{abstract}

\section{Introduction:}

Approximately, one third of patients with heart failure and reduced ejection fraction (EF) have left bundle branch block (LBBB $)^{4}$. Clark et $\mathrm{al}^{5}$ have shown that this proportion increased from $34.0 \%$ at baseline to $36.7 \%, 37.7 \%$ and $42.3 \%$ at 1,2 and 3 years follow up, respectively. Baseline LBBB was associated with a worse outcome and development of new LBBB was an independent adverse prognostic feature. CRT with BiVP plays an important role in the management of HF patient with LBBB. By reestablishing synchrony between the left and right ventricle, BIV pacing improves clinical parameters (NYHA class, 6-minute walk test, quality-of-life, and hospitalization rate) and echocardiographic indicators such as LVEF, LV end diastolic and diastolic volumes ${ }^{6}$. Importantly, CRT with BIV pacing trials with ${ }^{7}$ or without ICD $^{8}$ decreases hospitalization and mortality. Furthermore, patients with narrowing of the QRS on ECG with BIV pacing had a better survival rate and rapidly recovering left ventricular systolic function ${ }^{9}$. New pacing modalities for CRT are being assessed in clinical trials. HBP pacing has been shown to circumvent proximal LBBB and restore electrical resynchronization in patients with HF. LVSP has emerged as an alternative method for delivering CRT particularly in patients with infranodal atrioventricular block and LBBB. In this review article, we will discuss the options of CRT in HF and LBBB with BiVP, HBP and LVSP.

In LBBB, the onset of electrical activation occurs in the RV and then slowly propagates through the interventricular septum towards the lateral wall of the $\mathrm{LV}^{10}$. In patients with $\mathrm{HF}$ and LBBB, LV endocardial breakthrough is heterogenous and may occur at different septal regions ${ }^{11,12}$. . Auricchio et al have demonstrated that patients with LBBB morphology have a specific "U-shaped" activation sequence that turns around the apex and inferior wall of the LV. This activation pattern is generated by a functional line of block that is oriented from the base toward the apex of the $\mathrm{LV}^{11}$. The altered electrical activation of the ventricles results in a significant delay between the onset of LV and RV contraction ${ }^{13}$. This dyssynchrony results in reduction of LVEF and is associated with decrease in cardiac output and mean arterial pressure ${ }^{14,15}$. In addition, LBBB induced dyssynchrony causes redistribution of circumferential shortening and myocardial blood flow and that leads to LV remodeling ${ }^{16}$. 
CRT restores coordinated contraction and improves net systolic performance within one beat ${ }^{17}$, and increases ejection. Interestingly, this is achieved without a rise in myocardial oxygen consumption ${ }^{18,19}$. Moreover, Kyriacou et al. have shown that CRT improves coronary blood flow and flow velocity predominantly by increasing the dominant diastolic backward decompression (suction) wave ${ }^{20}$.

\section{Clinical trials in CRT :}

To date, more than 4000 patients have been enrolled in randomized controlled trials for CRT. Benefits have been demonstrated for patients with New York Heart Association (NYHA) class III HF, in particular, and, to some degree, for those with class IV HF. These trials have demonstrated consistent improvements in quality of life, functional status, exercise capacity and mortality, with the weight of evidence supporting current practice and guideline recommendations ${ }^{21}$.

\section{BIV pacing in moderate-to-severe $\mathrm{HF}$}

For patients with moderate-to-severe HF (NYHA class III- IV), CRT has been shown in 4 trials (MUSTIC SR, MIRACLE, CONTAK-CD, MIRACLEICD ${ }^{1,2,22,23}$ ) to provide improvements in NYHA functional class, exercise capacity (measured by the 6-minute hall walk distance) and peak oxygen consumption and quality of life.

The CARE-HF study randomized patients to either CRT plus optimal medical therapy or CRT alone. It was the first trial showing that CRT without an ICD prolonged survival and reduced both morbidity and mortality, with the effect sustained over 37 months of follow up ${ }^{24}$. Similarly, in the COMPANION trial, Bristow et al have shown that BiVP decreased the combined risk of death from any cause or first hospitalization and, when combined with an implantable cardioverted defibrillator (ICD), significantly reduces mortality ${ }^{7}$. A meta-analysis of 6 randomized, controlled trials of CRT for more severe, chronic, symptomatic LV systolic dysfunction found that CRT was associated with a $28 \%$ reduction in all-cause mortality and a $37 \%$ reduction in new hospitalizations for exacerbation of $\mathrm{HF}^{25}$.

In the COMPANION trial, patients with advanced heart failure (NYHA III or IV) due to ischemic or nonischemic cardiomyopathies and a QRS interval of at least $120 \mathrm{msec}$ were randomly assigned to receive optimal pharmacologic therapy alone or in combination with CRT with either a pacemaker or a pacemakerdefibrillator. The primary composite end point was the time to death from or hospitalization for any cause. As compared with optimal pharmacologic therapy alone, CRT with a pacemaker or a defibrillator significantly decreased the risk of the primary end point. The risk of the combined end point of death from or hospitalization for heart failure was also significantly reduced in the pacemaker and the defibrillator groups ${ }^{7}$. The CARE-HR trial enrolled patients with NYHA class III or IV heart failure due to left ventricular systolic dysfunction and cardiac dyssynchrony to receive standard pharmacologic therapy alone or with cardiac resynchronization. The primary end point was the time to death from any cause or an unplanned hospitalization for a major cardiovascular event. The principal secondary end point was death from any cause. As compared with medical therapy alone, CRT significantly decreased the risk of the primary endpoints. Also, CRT significantly reduced the interventricular mechanical delay, the end-systolic volume index, and the area of the mitral regurgitant jet; increased the left ventricular ejection fraction; and improved symptoms and the quality of life ${ }^{8}$.

\section{BIV pacing in less severe $\mathrm{HF}$}

While the benefits of CRT in moderate to severe HF patients has been well established, it has been more controversial in less severely ill HF patients (NYHA class II or even I). A series of randomized clinical trials (REVERSE ${ }^{26}$, MADIT-CRT ${ }^{27}$, Resynchronization/Defibrillation for Ambulatory Heart Failure Trial $[\mathrm{RAFT}]^{28}$ ) have enrolled nearly 5000 patient with NYHA I-II heart failure.

In MADIT-CRT, patients in NYHA I or II and an LVEF less than or equal $30 \%$ with either LBBB, RBBB or nonspecific intraventricular conduction delay were randomized to either an implantable cardioverter defibrillator (ICD) or CRT with a defibrillator. The hazard ratios for the primary end point (a composite of death from any cause and nonfatal HF-related adverse events) for comparisons of CRT-D patients versus 
patients who only received an ICD were significantly lower in LBBB patients than in non-LBBB patients. The risk of ventricular tachycardia, ventricular fibrillation, or death was decreased significantly in CRT-D patients with LBBB but not in non-LBBB patients. Echocardiographic parameters showed significantly greater reduction in left ventricular volumes and increase in ejection fraction with CRT-D in LBBB than in non-LBBB patients.

In the REVERSE trial, patients who received CRT pacemakers or defibrillators, with QRS more than or equal $120 \mathrm{~ms}$ and LV ejection fraction less than or equal $40 \%$ were assigned to active (CRT ON) versus control (CRT OFF) treatment. All patients were in sinus rhythm and receiving optimal medical therapy. The primary study end point was the proportion worsened by the heart failure (HF) clinical composite response. Compared with those of control subjects, clinical outcomes and LV function were improved and LV dimensions were decreased.

The RAFT trial, randomly assigned patients with New York Heart Association (NYHA) class II or III heart failure, a left ventricular ejection fraction of $30 \%$ or less, and an intrinsic QRS duration of 120 msec or more or a paced QRS duration of $200 \mathrm{msec}$ or more to receive either an ICD alone or an ICD plus CRT. The primary outcome was death from any cause or hospitalization for heart failure. A significantly less primary outcome occurred in the ICD-CRT group compared with the ICD group.

\section{Benefit of CRT:}

It is not completely understood how cardiac resynchronization therapy improves mechanical LV function in patients with heart failure and LBBB. Electrical resynchronization can reduce the LBBB-induced mechanical interventricular dyssynchrony between the right and the left ventricle and the intraventricular dyssynchrony within the left ventricle. Minimizing dyssynchrony has been shown to improve global LV function, increase LV filling time, decrease septal discordinated activity and reduce mitral regurgitation, thus improving hemodynamic ${ }^{17,29}$. To assess the impact of CRT on LV remodeling, three CRT studies have been conducted. In these studies, serial transthoracic Doppler echocardiography were used to assess reverse LV remodeling in advanced systolic heart failure. Patients were randomized to receive CRT with optimal medical therapy or medical therapy alone ${ }^{1,30}$. CRT resulted in a significant decrease in LV size, assessed as LV end-diastolic and end-systolic diameters or as LV volumes as early as $1 \mathrm{month}^{8,31,32}$, compared with control patients. There is further progressive reduction in LV diameter and LV volume at 6 months, which are sustained at 1 year in 65-75\% of patients ${ }^{33-35}$. The progressive reduction in LV volume with CRT is associated with restoration of mitral valve annular diameter and mitral subvalvular geometry towards normal. The changes in LV cavity shape and geometry of the mitral valve apparatus are associated with reduction in the severity of mitral regurgitation. In the MIRACLE study, the severity of mitral regurgitation had decreased significantly with $\mathrm{CRT}$ at 3 months, and this improvement was maintained at 6 and 12 months $^{36}$. There is some evidence that the decrease in the severity of mitral regurgitation precedes the reduction in LV volume and the associated changes in LV and mitral valve apparatus architecture ${ }^{37}$. Reverse LV remodeling requires continuous CRT. This was demonstrated clearly when CRT was discontinued after 3 months in one small open-label study ${ }^{37}$. After 3 months of CRT-induced reverse LV remodeling, cessation of CRT resulted in rapid abolition of the LV volume reduction with concomitant recurrent LV dilatation, progressive deterioration in LVEF and recurrent mitral regurgitation.

\section{Non responders to BIV pacing}

The most important question to address about non response to CRT is how to define non responders. Some studies define non responders based on absence of LV remodeling, whereas other studies define non responders based on lack of symptom improvement.

Moreover, achievement of hemodynamic stability, even in the presence of persistent dyspnea or absence of significant reverse remodeling, as demonstrated by Mullens et $\mathrm{al}^{38}$, might be also a desirable goal of CRT. Definition of response to CRT can also be challenged by patients' expectations. Patients in advanced heart failure are looking for symptoms' relief and as they feel significantly better after CRT, their attention would be directed toward less frequent admission to the hospital, greater need for social life and activities, and 
finally prolongation of life ${ }^{39}$.

Assessment of CRT response

$\mathrm{Yu}$ et $\mathrm{al}^{40}$ first demonstrated that a better outcome is achieved with a reduction of left ventricular (LV) end-systolic volume (ESV) of at least $10 \%$ after CRT; patients who showed a significant reverse remodeling had survival rate close to $90 \%$ at 3 year follow-up compared to a survival rate of about $50 \%$ of the remaining patients. Similarly, Ypenburg et $\mathrm{al}^{41}$, showed that both hospitalization and mortality were related to the magnitude of change in LVESV; patients who showed further dilatation of LVESV had the highest event rate (about $70 \%$ at 3 years follow-up) compared to those who showed impressive reduction (near normalization) of LVESV (about 6\%). It needs to be kept in mind that measuring reverse remodeling has several technical problems. The assessment of LV volume and LVEF using 2-dimensional echocardiography (the most frequently used technique) has a relatively large inter-observer variability ${ }^{39}$. The Predictors of Response to CRT (PROSPECT) study used 2-dimensional echocardiography for quantifying LV volumes ${ }^{42}$; the coefficient of variation for LVESV measurement was $3.8 \%$ for intra-observer variability but was as high as $14.5 \%$ for inter-observer variability.

Predictors of CRT Response

Patients with LBBB morphology have demonstrated the best response to CRT, whereas those with nonLBBB morphology generally have responded poorly. In a meta-analysis of COMPANION, CARE-HF, RAFT, and MADIT-CRT, Sipahi et al. ${ }^{43}$ found that patients with RBBB or IVCD did not benefit from CRT (relative risk for the composite primary outcome: $0.97 ; 95 \%$ confidence interval: 0.82 to $1.15 ; \mathrm{p}=0.75$ ).

Birnie et al. ${ }^{44}$ analysed data from the RAFT trial. A total of 1,483patients in sinus rhythm with QRS durations $>120 \mathrm{~ms}$ were examined. A LBBB was present in 1,175 patients (79.2\%), 141 patients had an RBBB (9.5\%), and the remainder (11.3\%) had an IVCD. Patients with RBBB and IVCD were more likely to have an ischemic etiology of HF. Among the patients with LBBB, the benefit of CRT increased directly as QRS duration increased. In contrast, the benefit of CRT only began to emerge in the non-LBBB patients once the QRS duration was $>160 \mathrm{~ms}$ and only after 2 years of follow-up. Sundaram V et al. ${ }^{45}$ has shown from the analysis of the Medicare registry that the improvements in both survival and HF hospitalization with CRT-D were greatest in patients with QRSD [?]180 ms with or without LBBB. The presence of electrical resynchronization leading eventually to mechanical resynchronization predicts a good response to CRT. This can be determined by observing the reduction in QRS duration ${ }^{46}$ and by a change in the shape of the QRS complex (indicating fusion of right ventricular and LV originating activation waves) ${ }^{47}$. A sequence of right-to-left electrical and mechanical activation that is present in LBBB or RV pacing is detrimental ${ }^{11,17,48}$. Thus, proper fusion of RV and LV activation waves requires proper positioning of the pacing leads. Also, the amount of scar tissue appears to be a predictor of CRT-non-response ${ }^{49}$. Bleeker et al. have shown that CRT does not reduce LV dyssynchrony in patients with transmural scar tissue in the posterolateral LV segments, resulting in clinical and echocardiographic nonresponse to CRT.

Considering that location of the pacing lead is of paramount importance, it is preferred for the LV lead to be placed in non-apical areas ${ }^{50}$. A posterolateral scar should be avoided and the areas of maximal electrical delay should be targeted ${ }^{51}$. Patients are more likely to be responders if the $\mathrm{qLV}$ at implant is more than 95 $\mathrm{ms}^{52}$. Alternatively, , LV can be paced from more than one site either by multipoint pacing (pacing from multiple sites from the same lead) or from multisite LV pacing ${ }^{53}$. In multisite LV pacing, the first LV lead is inserted into a postero-lateral or lateral vein. The second LV lead is placed as far as possible from the first lead, in the anterior vein, a high antero-lateral vein, or the middle cardiac vein. Another modality for LV pacing is LV endocardial pacing with transventricular delivery of an active-fixation lead to the endocardial wall of the lateral $L V{ }^{54}$. Advantage of LV endocardial pacing is easy access to all LV endocardial surface but the disadvantages are requirement for lifelong anticoagulation and the risk for lead extraction. One interesting new technique is the LV pellet that can be delivered retrogradely via the aorta into an LV site. In the select LV study, Reddy et al. applied this technology to non-responders and showed an improvement of EF at 6 months ${ }^{55}$. 


\section{Role of His pacing: Better option than BiV pacing?}

His bundle pacing (HBP) has been considered as an alternative to RV pacing and in patients with bundle branch block with indication for BIV pacing or as a rescue strategy in failed LV lead implantation. It is frequently possible with HBP to recruit the native conducting system. The mechanisms for the reduction in QRS duration with HBP remain to be fully elucidated but may include recruitment of fibers distal to the site of delay, longitudinal dissociation, capture attributable to higher pacing outputs, and hyperpolarizing dormant His bundle tissue ${ }^{56}$. In the His Resynchronization Versus Biventricular Pacing in Patients With Heart Failure and Left Bundle Branch Block trial ${ }^{57}$, Arnold et al. have conducted a study on patients with heart failure and left bundle branch block referred for conventional BIV pacing and using noninvasive epicardial electrocardiographic imaging to identify patients in whom His bundle pacing shortened left ventricular activation time. In these patients, the authors compared the hemodynamic effects of His bundle pacing against biventricular pacing. In 18 of 23 patients, left ventricular activation time was significantly shortened by His bundle pacing. In the 17 patients who had a complete electromechanical dataset, His bundle pacing was more effective than biventricular pacing at delivering ventricular resynchronization: greater reduction in QRS duration (-18.6 ms; 95\% confidence interval [CI]: -31.6 to $-5.7 \mathrm{~ms} ; \mathrm{p}=0.007)$, left ventricular activation time (-26 ms; $95 \%$ CI: -41 to $-21 \mathrm{~ms} ; \mathrm{p}=0.002)$, and left ventricular dyssynchrony index $(-11.2 \mathrm{~ms} ; 95 \%$ CI: -16.8 to $-5.6 \mathrm{~ms} ; \mathrm{p}<0.001)$. His bundle pacing also produced a greater acute hemodynamic response ( $4.6 \mathrm{~mm} \mathrm{Hg} ; 95 \%$ CI: 0.2 to $9.1 \mathrm{~mm} \mathrm{Hg}$; p = 0.04). The authors concluded that His resynchronization delivers better ventricular resynchronization, and greater improvement in hemodynamic parameters, than biventricular pacing.

Lustgarten et $\mathrm{al}^{58}$, in his crossover comparison study between HBP and BiV pacing, HBP have shown that the effect of His pacing is equivalent to BIV pacing.

Twenty-nine patients were enrolled and were implanted with RA pacing lead, RV defibrillation lead, LV lead via the coronary sinus, and HBP lead. His and LV leads were plugged into the LV port via a Y-adapter. After successful implant, patients were randomized in single patient-blinded fashion to either HBP or BIV pacing. There was no difference between the two groups. After 6 months, patients were crossed over to the other pacing modality and followed for another 6 months. 21 patients (72\%) in the HIS pacing group demonstrated QRS narrowing at implant. Clinical outcomes (quality of life, New York Heart Association functional class, 6-minute hall walk test, LV ejection fraction) were significantly improved for both pacing modes compared with baseline measures. The HIS Sync trial ${ }^{59}$ is the first trial that compared His pacing to BIV pacing in patients with heart failure and LBBB. In this trial the average LVEF was $28 \%$, and QRS width $168+/-18$ ms with 35 patients having left bundle branch block pattern,2 patients with right bundle branch block and 3 patients with RV paced rhythm. 21 patients were randomized to His-CRT and 20 patients to $\mathrm{BiV}-\mathrm{CRT}$. Baseline characteristics were pretty much similar between the two groups except that LVEF was significantly lower among patients in the His-CRT group (median 26.3\%) compared with patients in the BiV group $(30.5 \%)$ with a $\mathrm{p}$ value of 0.011 . $48 \%$ of patients in the His-CRT group and $26 \%$ of patients in the $\mathrm{BiV}$ group crossed over. The most common reasons for crossover were inability to correct QRS width (5 patients) in the His pacing group and suboptimal venous anatomy (4 patients) in BiV group. Using intention-to-treat analysis, the QRS duration was significantly reduced with His pacing $(172+/-16 \mathrm{~ms}$ to $144+/-30 \mathrm{~ms} ; \mathrm{p}=$ $0.002)$ but not with $\mathrm{BiV}$ pacing $(165+-18 \mathrm{~ms}$ to $152+-30 \mathrm{~ms} ; \mathrm{p}=0.11)$. This difference was not significant when comparing both groups $(\mathrm{p}=0.42)$. There was similar improvement in LVEF at a median follow up of 6.2 months (26.3\% to $31.9 \%$; $<0.001$ in the His pacing group) and (30.5\% to $34.0 \% ; \mathrm{p}<0.001$ in the BIV pacing group). The HIS SYNC trial did not show any difference between HIS pacing and BIV pacing in terms of electrical and echocardiographic parameters keeping in mind that the cross over rate was high and that could have impacted treatment efficacy.

\section{Role of Left Bundle Branch Pacing: will it replace other pacing modality for CRT?}

LVSP has emerged as an alternative method for delivering physiological pacing particularly in patients with infranodal atrioventricular block and left bundle branch block (LBBB). Though HBP can correct classic LBBB in $97 \%$ of patients ${ }^{60}$, the site of block is often located within the His or proximal left bundle ${ }^{61}$. 
The proximal left bundle branches run through the LV septum and fan out proving a wider target for pacing. Huang et al have reported a technique for left bundle pacing using a transseptal approach ${ }^{62}$. Left bundle pacing has been reported to have low pacing thresholds, larger $\mathrm{R}$ waves and, by targeting the distal conduction system, a lower theoretical risk for developing distal conduction block ${ }^{63}$. The LVSP could be an attractive way to overcome the majority of the limitations of HBP. These include difficulty in identifying the adequate location of the His bundle, high and unstable threshold in up to $10 \%$ of patients, damage to the His bundle during implantation, heart block distal to the His bundle, undersensing $\mathrm{R}$ wave amplitude and oversensing atrial signals and lower success rate in patients with left bundle branch block (LBBB) and heart failure due to high pacing output required to correct $\mathrm{LBBB}^{58,60,64-68}$. The rationale behind correcting LBBB by HBP is that increased pacing output captures the left bundle branch (LBB) beyond the area of block. El-Sherif et al. ${ }^{69}$ showed that HBP required $20 \mathrm{~V}$ to capture the LBB. Huang et al. ${ }^{62}$ first reported direct left bundle branch pacing in patients with LBBB and heart failure. $\mathrm{HBP}$ at $10 \mathrm{~V}$ failed to correct LBBB but after advancing the pacing tip towards the left ventricle LBBB resolved at a low pacing capture threshold $(0.5 \mathrm{~V})$. The heart failure symptoms improved, $\mathrm{LV}$ ejection fraction improved and adjustment of pacing parameters led to normalization of QRS. Chen et al. ${ }^{70}$ used a transventricular septal approach for left bundle pacing in patients with bradycardia. In their study, ECG configuration changing from LBBB pattern to RBBB pattern was observed as the pacing tip was advanced from the right interventricular septum to capture the left bundle branch. Demonstration of left bundle potential during intrinsic rhythm provided strong evidence of successful left bundle pacing. Notably, paced ECG QRS duration was significantly shorter $(111.85 \pm 10.77 \mathrm{~ms})$ compared with either RV septal pacing $(154.80 \pm 9.85 \mathrm{~ms})$ or RV apical pacing (165.50 $\pm 17.80 \mathrm{~ms})$ with comparable capture threshold. Interestingly, both left and right bundle branch block could be corrected by left bundle pacing at low capture threshold. Interestingly, both left and right bundle branch block could be corrected by LVSP at a low capture threshold. Additionally, the QRS in LVSP is typically $<130 \mathrm{~ms}$ instead of complete RBBB. It is also significantly shorter than during RV pacing ${ }^{70,71}$. This may be explained by retrograde activation of the right bundle during pacing or connection between the main right and left bundle branches ${ }^{72,73}$ or cell to cell conduction ${ }^{74}$. The LVSP procedural success is $80.5-93 \%$ in small studies ${ }^{71,75,76}$. Vijayaraman et $\mathrm{al}^{76}$. reported periprocedural lead dislodgement in 3 of 97 patients who underwent LVSP. Potential complications for left bundle pacing procedure include right bundle branch (RBB) injury during the procedure, lead dislodgement, septal perforation and coronary artery injury especially the septal perforators ${ }^{77}$.

\section{Conclusion}

BiV pacing has shown to improve symptoms and reduce hospitalizations and mortality in large randomized controlled trials and remains the standard of care for patients with HF and significant electrical dysynchrony. New modalities such as HBP and LVSP has shown to be equal or superior to BiV pacing in regards to the surrogate measures such as improvement in hemodynamics as well as with LV remodeling. There are no large clinical trials comparing BiV pacing with other pacing modalities such as HBP and LVSP for hard efficacy endpoints such as hospitalization and mortality and are unlikely to be performed in the future. Currently, HBP and LVSP has an important role to play in patients who are unable to receive BiV pacing for one reason or the other. Future of HBP and LVSP looks promising and only time will tell if it will replace the conventional BiV pacing.

\section{References:}

1. Abraham WT, Fisher WG, Smith AL, et al. Cardiac Resynchronization in Chronic Heart Failure. N Engl J Med 2002;346(24):1845-53.

2. Cazeau S, Leclercq C, Lavergne T, et al. Effects of Multisite Biventricular Pacing in Patients with Heart Failure and Intraventricular Conduction Delay. N Engl J Med 2001;344(12):873-80.

3. Fonarow GC, Albert NM, Curtis AB, et al. Incremental Reduction in Risk of Death Associated With Use of Guideline-Recommended Therapies in Patients With Heart Failure: A Nested CaseControl Analysis of IMPROVE HF. JAHA [Internet] 2012 [cited 2020 Apr 18];1(1). Available from: 
https://www.ahajournals.org/doi/10.1161/JAHA.111.000018

4. Francia P, Balla C, Paneni F, Volpe M. Left bundle-branch block-pathophysiology, prognosis, and clinical management. Clin Cardiol 2007;30(3):110-5.

5. Clark AL, Goode K, Cleland JGF. The prevalence and incidence of left bundle branch block in ambulant patients with chronic heart failure. European Journal of Heart Failure 2008;10(7):696-702.

6. Bleeker GB, Mollema SA, Holman ER, et al. Left Ventricular Resynchronization Is Mandatory for Response to Cardiac Resynchronization Therapy: Analysis in Patients With Echocardiographic Evidence of Left Ventricular Dyssynchrony at Baseline. Circulation 2007;116(13):1440-8.

7. Bristow MR, Saxon LA, Boehmer J, et al. Cardiac-Resynchronization Therapy with or without an Implantable Defibrillator in Advanced Chronic Heart Failure. N Engl J Med 2004;350(21):2140-50.

8. Cleland JGF, Daubert J-C, Erdmann E, et al. The Effect of Cardiac Resynchronization on Morbidity and Mortality in Heart Failure. N Engl J Med 2005;352(15):1539-49.

9. Gasparini M, Mantica M, Galimberti P, et al. Beneficial Effects of Biventricular Pacing in Patients with a "Narrow" QRS. Pacing and Clinical Electrophysiology 2003;26(1p2):169-74.

10. Vassallo JA, Cassidy DM, Marchlinski FE, et al. Endocardial activation of left bundle branch block. Circulation 1984;69(5):914-23.

11. Auricchio A, Fantoni C, Regoli F, et al. Characterization of Left Ventricular Activation in Patients With Heart Failure and Left Bundle-Branch Block. Circulation 2004;109(9):1133-9.

12. Rodriguez L-M, Timmermans C, Nabar A, Beatty G, Wellens HJJ. Variable Patterns of Septal Activation in Patients with Left Bundle Branch Block and Heart Failure: Septal Activation in Patients with LBBB and Heart Failure. Journal of Cardiovascular Electrophysiology 2003;14(2):135-41.

13. Grines CL, Bashore TM, Boudoulas H, Olson S, Shafer P, Wooley CF. Functional abnormalities in isolated left bundle branch block. The effect of interventricular asynchrony. Circulation 1989;79(4):845-53.

14. Bramlet DA, Morris KG, Coleman RE, Albert D, Cobb FR. Effect of rate-dependent left bundle branch block on global and regional left ventricular function. Circulation 1983;67(5):1059-65.

15. Takeshita A, Basta LL, Kioschos JM. Effect of intermittent left bundle branch block on left ventricular performance. The American Journal of Medicine 1974;56(2):251-5.

16. Vernooy K, Verbeek XAAM, Peschar M, et al. Left bundle branch block induces ventricular remodelling and functional septal hypoperfusion. European Heart Journal 2005;26(1):91-8.

17. Kass DA, Chen C-H, Curry C, et al. Improved Left Ventricular Mechanics From Acute VDD Pacing in Patients With Dilated Cardiomyopathy and Ventricular Conduction Delay. Circulation 1999;99(12):1567-73.

18. Ukkonen H, Beanlands RSB, Burwash IG, et al. Effect of Cardiac Resynchronization on Myocardial Efficiency and Regional Oxidative Metabolism. Circulation 2003;107(1):28-31.

19. Nelson GS, Berger RD, Fetics BJ, et al. Left Ventricular or Biventricular Pacing Improves Cardiac Function at Diminished Energy Cost in Patients With Dilated Cardiomyopathy and Left Bundle-Branch Block. Circulation 2000;102(25):3053-9.

20. Kyriacou A, Whinnett ZI, Sen S, et al. Improvement in Coronary Blood Flow Velocity With Acute Biventricular Pacing Is Predominantly Due to an Increase in a Diastolic Backward-Travelling Decompression (Suction) Wave. Circulation 2012;126(11):1334-44.

21. Linde C, Ellenbogen K, McAlister FA. Cardiac resynchronization therapy (CRT): Clinical trials, guidelines, and target populations. Heart Rhythm 2012;9(8):S3-13. 
22. Higgins SL, Hummel JD, Niazi IK, et al. Cardiac resynchronization therapy for the treatment of heart failure in patients with intraventricular conduction delay and malignant ventricular tachyarrhythmias. Journal of the American College of Cardiology 2003;42(8):1454-9.

23. Abraham WT, Young JB, Leon AR, et al. Effects of Cardiac Resynchronization on Disease Progression in Patients With Left Ventricular Systolic Dysfunction, an Indication for an Implantable CardioverterDefibrillator, and Mildly Symptomatic Chronic Heart Failure. Circulation 2004;110(18):2864-8.

24. Cleland JGF, Daubert J-C, Erdmann E, et al. Longer-term effects of cardiac resynchronization therapy on mortality in heart failure [the CArdiac REsynchronization-Heart Failure (CARE-HF) trial extension phase]. European Heart Journal 2006;27(16):1928-32.

25. Rossi A, Rossi G, Piacenti M, Startari U, Panchetti L, Morales M-A. The current role of cardiac resynchronization therapy in reducing mortality and hospitalization in heart failure patients: a meta-analysis from clinical trials. Heart Vessels 2008;23(4):217-23.

26. Daubert C, Gold MR, Abraham WT, et al. Prevention of Disease Progression by Cardiac Resynchronization Therapy in Patients With Asymptomatic or Mildly Symptomatic Left Ventricular Dysfunction. Journal of the American College of Cardiology 2009;54(20):1837-46.

27. Zareba W, Klein H, Cygankiewicz I, et al. Effectiveness of Cardiac Resynchronization Therapy by QRS Morphology in the Multicenter Automatic Defibrillator Implantation Trial-Cardiac Resynchronization Therapy (MADIT-CRT). Circulation 2011;123(10):1061-72.

28. Tang ASL, Wells GA, Talajic M, et al. Cardiac-Resynchronization Therapy for Mild-to-Moderate Heart Failure. N Engl J Med 2010;363(25):2385-95.

29. Auricchio A, Stellbrink C, Block M, et al. Effect of Pacing Chamber and Atrioventricular Delay on Acute Systolic Function of Paced Patients With Congestive Heart Failure. Circulation 1999;99(23):2993-3001.

30. St John Sutton MG, Plappert T, Abraham WT, et al. Effect of Cardiac Resynchronization Therapy on Left Ventricular Size and Function in Chronic Heart Failure. Circulation 2003;107(15):1985-90.

31. Molhoek SG, Bax JJ, van Erven L, et al. Comparison of benefits from cardiac resynchronization therapy in patients with ischemic cardiomyopathy versus idiopathic dilated cardiomyopathy. The American Journal of Cardiology 2004;93(7):860-3.

32. Linde C, Leclercq C, Rex S, et al. Long-term benefits of biventricular pacing in congestive heart failure: results from the MUltisite STimulation in cardiomyopathy (MUSTIC) study. Journal of the American College of Cardiology 2002;40(1):111-8.

33. Burns RJ, Gibbons RJ, Yi Q, et al. The relationships of left ventricular ejection fraction, end-systolic volume index and infarct size to six-month mortality after hospital discharge following myocardial infarction treated by thrombolysis. Journal of the American College of Cardiology 2002;39(1):30-6.

34. Chuang ML, Hibberd MG, Salton CJ, et al. Importance of imaging method over imaging modality in noninvasive determination of left ventricular volumes and ejection fraction. Journal of the American College of Cardiology 2000;35(2):477-84.

35. St John Sutton M, Pfeffer MA, Plappert T, et al. Quantitative two-dimensional echocardiographic measurements are major predictors of adverse cardiovascular events after acute myocardial infarction. The protective effects of captopril. Circulation 1994;89(1):68-75.

36. St John Sutton MG, Plappert T, Hilpisch KE, Abraham WT, Hayes DL, Chinchoy E. Sustained Reverse Left Ventricular Structural Remodeling With Cardiac Resynchronization at One Year Is a Function of Etiology: Quantitative Doppler Echocardiographic Evidence From the Multicenter InSync Randomized Clinical Evaluation (MIRACLE). Circulation 2006;113(2):266-72. 
37. Yu C-M, Chau E, Sanderson JE, et al. Tissue Doppler Echocardiographic Evidence of Reverse Remodeling and Improved Synchronicity by Simultaneously Delaying Regional Contraction After Biventricular Pacing Therapy in Heart Failure. Circulation 2002;105(4):438-45.

38. Mullens W, Verga T, Grimm RA, Starling RC, Wilkoff BL, Tang WHW. Persistent Hemodynamic Benefits of Cardiac Resynchronization Therapy With Disease Progression in Advanced Heart Failure. Journal of the American College of Cardiology 2009;53(7):600-7.

39. Auricchio A, Prinzen FW. Non-Responders to Cardiac Resynchronization Therapy. Circ J 2011;75(3):521-7.

40. Yu C-M, Bleeker GB, Fung JW-H, et al. Left Ventricular Reverse Remodeling but Not Clinical Improvement Predicts Long-Term Survival After Cardiac Resynchronization Therapy. Circulation 2005;112(11):1580-6.

41. Ypenburg C, van Bommel RJ, Borleffs CJW, et al. Long-Term Prognosis After Cardiac Resynchronization Therapy Is Related to the Extent of Left Ventricular Reverse Remodeling at Midterm Follow-Up. Journal of the American College of Cardiology 2009;53(6):483-90.

42. Chung ES, Leon AR, Tavazzi L, et al. Results of the Predictors of Response to CRT (PROSPECT) Trial. Circulation 2008;117(20):2608-16.

43. Sipahi I, Chou JC, Hyden M, Rowland DY, Simon DI, Fang JC. Effect of QRS morphology on clinical event reduction with cardiac resynchronization therapy: Meta-analysis of randomized controlled trials. American Heart Journal 2012;163(2):260-267.e3.

44. Birnie DH, Ha A, Higginson L, et al. Impact of QRS Morphology and Duration on Outcomes After Cardiac Resynchronization Therapy: Results From the Resynchronization-Defibrillation for Ambulatory Heart Failure Trial (RAFT). Circ Heart Fail 2013;6(6):1190-8.

45. Sundaram V, Sahadevan J, Waldo AL, et al. Implantable Cardioverter-Defibrillators With Versus Without Resynchronization Therapy in Patients With a QRS Duration >180 ms. Journal of the American College of Cardiology 2017;69(16):2026-36.

46. Rademakers LM, van Kerckhoven R, van Deursen CJM, et al. Myocardial Infarction Does Not Preclude Electrical and Hemodynamic Benefits of Cardiac Resynchronization Therapy in Dyssynchronous Canine Hearts. Circ Arrhythm Electrophysiol 2010;3(4):361-8.

47. Seo Y, Ito H, Nakatani S, et al. The Role of Echocardiography in Predicting Responders to Cardiac Resynchronization Therapy. Circ J 2011;75(5):1156-63.

48. Prinzen FW, Hunter WC, Wyman BT, McVeigh ER. Mapping of regional myocardial strain and work during ventricular pacing: experimental study using magnetic resonance imaging tagging. Journal of the American College of Cardiology 1999;33(6):1735-42.

49. Bleeker GB, Kaandorp TAM, Lamb HJ, et al. Effect of Posterolateral Scar Tissue on Clinical and Echocardiographic Improvement After Cardiac Resynchronization Therapy. Circulation 2006;113(7):96976.

50. Singh JP, Klein HU, Huang DT, et al. Left Ventricular Lead Position and Clinical Outcome in the Multicenter Automatic Defibrillator Implantation Trial-Cardiac Resynchronization Therapy (MADIT-CRT) Trial. Circulation 2011;123(11):1159-66.

51. Varma N. Managing Non-responders Today and What's on the Horizon. JICRM 2016;7(00):S12-8.

52. Varma N, O'Donnell D, Bassiouny M, et al. Programming Cardiac Resynchronization Therapy for Electrical Synchrony: Reaching Beyond Left Bundle Branch Block and Left Ventricular Activation Delay. JAHA [Internet] 2018 [cited 2020 May 18];7(3). Available from: https://www.ahajournals.org/doi/10.1161/JAHA.117.007489 
53. Leclercq C, Gadler F, Kranig W, et al. A Randomized Comparison of Triple-Site Versus Dual-Site Ventricular Stimulation in Patients With Congestive Heart Failure. Journal of the American College of Cardiology 2008;51(15):1455-62.

54. Betts TR, Gamble JHP, Khiani R, Bashir Y, Rajappan K. Development of a Technique for Left Ventricular Endocardial Pacing via Puncture of the Interventricular Septum. Circ Arrhythm Electrophysiol 2014;7(1):17-22.

55. Reddy VY, Miller MA, Neuzil P, et al. Cardiac Resynchronization Therapy With Wireless Left Ventricular Endocardial Pacing. Journal of the American College of Cardiology 2017;69(17):2119-29.

56. Teng AE, Massoud L, Ajijola OA. Physiological mechanisms of QRS narrowing in bundle branch block patients undergoing permanent His bundle pacing. Journal of Electrocardiology 2016;49(5):644-8.

57. Arnold AD, Shun-Shin MJ, Keene D, et al. His Resynchronization Versus Biventricular Pacing in Patients With Heart Failure and Left Bundle Branch Block. Journal of the American College of Cardiology $2018 ; 72(24): 3112-22$.

58. Lustgarten DL, Crespo EM, Arkhipova-Jenkins I, et al. His-bundle pacing versus biventricular pacing in cardiac resynchronization therapy patients: A crossover design comparison. Heart Rhythm 2015;12(7):154857.

59. Upadhyay GA, Vijayaraman P, Nayak HM, et al. His Corrective Pacing or Biventricular Pacing for Cardiac Resynchronization in Heart Failure. Journal of the American College of Cardiology 2019;74(1):1579 .

60. Huang W, Su L, Wu S, et al. Long-term outcomes of His bundle pacing in patients with heart failure with left bundle branch block. Heart 2019;105(2):137-43.

61. Upadhyay GA, Cherian T, Shatz DY, et al. Intracardiac Delineation of Septal Conduction in Left BundleBranch Block Patterns: Mechanistic Evidence of Left Intrahisian Block Circumvented by His Bundle Pacing. Circulation 2019;139(16):1876-88.

62. Huang W, Su L, Wu S, et al. A Novel Pacing Strategy With Low and Stable Output: Pacing the Left Bundle Branch Immediately Beyond the Conduction Block. Canadian Journal of Cardiology 2017;33(12):1736.e11736.e3.

63. Wu S, Su L, Wang S, Vijayaraman P, Ellenbogen KA, Huang W. Peri-left bundle branch pacing in a patient with right ventricular pacing-induced cardiomyopathy and atrioventricular infra-Hisian block. EP Europace 2019;21(7):1038-1038.

64. Sharma PS, Dandamudi G, Herweg B, et al. Permanent His-bundle pacing as an alternative to biventricular pacing for cardiac resynchronization therapy: A multicenter experience. Heart Rhythm 2018;15(3):41320.

65. Zanon F, Ellenbogen KA, Dandamudi G, et al. Permanent His-bundle pacing: a systematic literature review and meta-analysis. EP Europace 2018;20(11):1819-26.

66. Barba-Pichardo R, Morina-Vazquez P, Fernandez-Gomez JM, Venegas-Gamero J, Herrera-Carranza M. Permanent His-bundle pacing: seeking physiological ventricular pacing. Europace 2010;12(4):527-33.

67. Subzposh FA, Vijayaraman P. Long-Term Results of His Bundle Pacing. Cardiac Electrophysiology Clinics 2018;10(3):537-42.

68. Vijayaraman P, Chung MK, Dandamudi G, et al. His Bundle Pacing. Journal of the American College of Cardiology 2018;72(8):927-47.

69. El-Sherif N, Amay-Y-Leon F, Schonfield C, et al. Normalization of bundle branch block patterns by distal His bundle pacing. Clinical and experimental evidence of longitudinal dissociation in the pathologic 
his bundle. Circulation 1978;57(3):473-83.

70. Chen K, Li Y, Dai Y, et al. Comparison of electrocardiogram characteristics and pacing parameters between left bundle branch pacing and right ventricular pacing in patients receiving pacemaker therapy. EP Europace 2019;21(4):673-80.

71. Li Y, Chen K, Dai Y, et al. Left bundle branch pacing for symptomatic bradycardia: Implant success rate, safety, and pacing characteristics. Heart Rhythm 2019;16(12):1758-65.

72. James TN et al. Fine structure of the His bundle. Circulation 1971;44:9-28

73. Barba-Pichardo R, Morina-Vazquez P, Venegas-Gamero J, Maroto-Monserrat F, Cid-Cumplido M, Herrera-Carranza M. [Permanent His-bundle pacing in patients with infra-Hisian atrioventricular block]. Rev Esp Cardiol 2006;59(6):553-8.

74. Zhang S, Zhou X, Gold MR. Left Bundle Branch Pacing. Journal of the American College of Cardiology 2019;74(24):3039-49.

75. Zhang J, Wang Z, Cheng L, et al. Immediate clinical outcomes of left bundle branch area pacing vs conventional right ventricular pacing. Clin Cardiol 2019;clc.23215.

76. Vijayaraman P, Subzposh FA, Naperkowski A, et al. Prospective evaluation of feasibility and electrophysiologic and echocardiographic characteristics of left bundle branch area pacing. Heart Rhythm 2019;16(12):1774-82.

77. Huang W, Chen X, Su L, Wu S, Xia X, Vijayaraman P. A beginner's guide to permanent left bundle branch pacing. Heart Rhythm 2019;16(12):1791-6. 